\title{
From Real-Time 3D Echocardiography to Mitral Valve Finite Element Analysis: A Novel Modeling Approach
}

\author{
E Votta ${ }^{1}$, A Arnoldi ${ }^{1}$, M Stevanella ${ }^{1}$, F Veronesi $^{1}$, G Tamborini ${ }^{2}$, \\ F Alamanni ${ }^{2}$, EG Caiani ${ }^{1}$, A Redaelli ${ }^{1}$ \\ ${ }^{1}$ Politecnico di Milano, Milano, Italy \\ ${ }^{2}$ Centro Cardiologico Monzino IRCCS, Milano, Italy
}

\begin{abstract}
Finite element models are an innovative tool for the biomechanical analysis of dynamic cardiac structures, such as the mitral valve. Still, existing models are limited by a simplified description of valve morphology.

We aimed at overcoming such limitation by integrating into a mitral valve structural finite element model the information about annulus and papillary muscles geometry and dynamics, obtained in humans by analysis of real time $3 D$ echocardiographic images.

The model was used to simulate valve closure from end diastole to systolic peak and to analyze the valve biomechanics. Simulated valvular dynamics and leaflets coaptation were realistic, and all gathered quantitative results were consistent with experimental findings from literature.

This novel approach may lead to the development of a patient-specific modeling tool for clinical purposes.
\end{abstract}

\section{Introduction}

The mitral valve (MV) is a complex apparatus consisting of two leaflets, inserted on the valvular plane through the mitral annulus (MA) and connected to the ventricular myocardium through a net of branched chordae tendineae that converge into two papillary muscles (PMs). The MV guarantees the unidirectional flow from the left atrium to the left ventricle during diastole and prevents backward flows during systole. Its function is driven by several factors: transvalvular pressure drop, dynamic contraction of MA and PMs and ventricular hemodynamics.

In the last decade, the high prevalence of MV pathologies, which require surgical intervention, has raised the need for more effective surgical techniques and devices, whose conceiving and application need careful design and testing. Finite element models (FEMs) are an innovative and helpful tool to be used in such process. Indeed, FEMs have the potential to quantitatively analyze MV biomechanics, with great benefits as compared to traditional animal models: absence of ethical issues, local quantification of mechanical parameters, control on the multiple factors leading the behaviour of the simulated system and, thus, the capability to answer "what if" questions. Thanks to such potential, FEMs have been recently applied to study MV normal function [1-3], the biomechanics underlying MV diseases [1] and effects of surgical corrections [4-7]. However, none of the mentioned studies captures all of the four aspects that drive MV function: morphology, tissues mechanical response, dynamic boundary conditions and interaction between the MV and surrounding blood. In particular, current FEMs, based on animal or ex vivo data, assume an idealized, symmetrical valvular structure and neglect the dynamic contraction of MA and PMs.

Real time 3-D echocardiography (RT3DE) offers the potential to non invasively assess MV structures over time, thus providing the information needed to overcome the abovementioned limitations.

Our aim was to integrate such quantitative information into a realistic FEM of the MV, which simulates valve closure from end diastole (ED) to systolic peak (SP).

\section{Methods}

\subsection{Real-time 3D ecocardiography}

Transthoracic RT3DE imaging was performed (iE33, Philips, Andover, MA) from the apical window using a fully sampled matrix-array transducer (X3), with the subject in the left lateral decubitus position. RT3DE datasets were acquired in a single normal subject (male, age 40) using the wide-angled mode at high frame rate $(31 \mathrm{~Hz})$, wherein 8 wedge-shaped subvolumes were obtained during 8 cardiac cycles during a single breath hold with ECG gating.

\subsection{RT3DE data analysis}

The RT3DE data were analyzed using previously developed custom software to semiautomatically detect and track the MA throughout the cardiac cycle, and to 
identify the PMs tips [8]. Briefly, on the ED frame, 18 long-axis cut planes evenly rotated around the center of the MA $\left(10^{\circ}\right.$ step $)$ were displayed to complete the initialization procedure. On each plane, the operator selected 2 points, one on each side of the MA. Subsequently, the positions of each initialized point were automatically tracked frame-by-frame in 3D space, using optical flow and region-based matching techniques. The automatically tracked points were displayed in each frame to verify their position, and manual corrections were performed when necessary. To identify the PMs tip, on the ED frame the dataset was analyzed by rotating the cut plane around the MA center, until the tip of each PM was best visualized. On the selected plane, the operator defined a point at the position of the PM tip. Moreover, on the ED frame additional information regarding leaflets orientation in space was gathered, measuring their tilting in an apical 4-chamber cut plane.
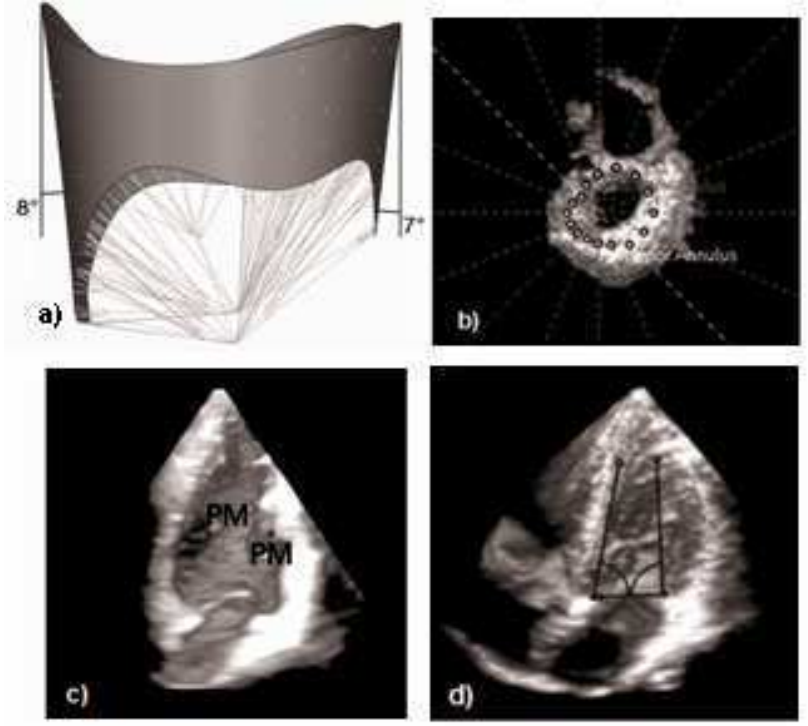

Figure 1: a) Valve geometrical model, b) Annular profile, c) papillary muscles and d) leaflets inclination are detected on the end diastolic ultrasound frame.

\subsection{MV geometrical model}

The ED configuration was chosen as the reference one. The MV geometrical model (Figure 1.a) was implemented defining i) the annular profile, by interpolation through $6^{\text {th }}$ order Fourier functions of the 36 points selected on the MA in the ED frame (Figure 1.b). The continuous profile was than sampled into 404 nodes that provided the seeding for the mesh to be defined on leaflets surface; ii) PMs tips, defined as the two points selected in the ED frame (Figure 1.c); iii) the leaflets, whose profile, based on ex-vivo data from the literature [9], was adapted to the subject's annular size and spatial orientation (Figure 1.d); iv) the chordal apparatus, consisting in 39 branched chordae of three orders: $1^{\text {st }}$ (marginal and commissural chordae), $2^{\text {nd }}$ (including strut chordae) and $3^{\text {rd }}$ (basal chordae). The number of chordae, the corresponding branched structure and insertion sites on the leaflets were defined in accordance to ex vivo findings [10]. A constant thickness of $1.32 \mathrm{~mm}$ and 1.26 $\mathrm{mm}$ was defined for anterior and posterior leaflet, respectively. Two transitional commissural zones with a thickness of $1.29 \mathrm{~mm}$ were also identified. Constant cross-section area values of $0.4,1.15$ and $0.79 \mathrm{~mm}^{2}$ were assigned to $1^{\text {st }}$ order, strut and basal chordae.

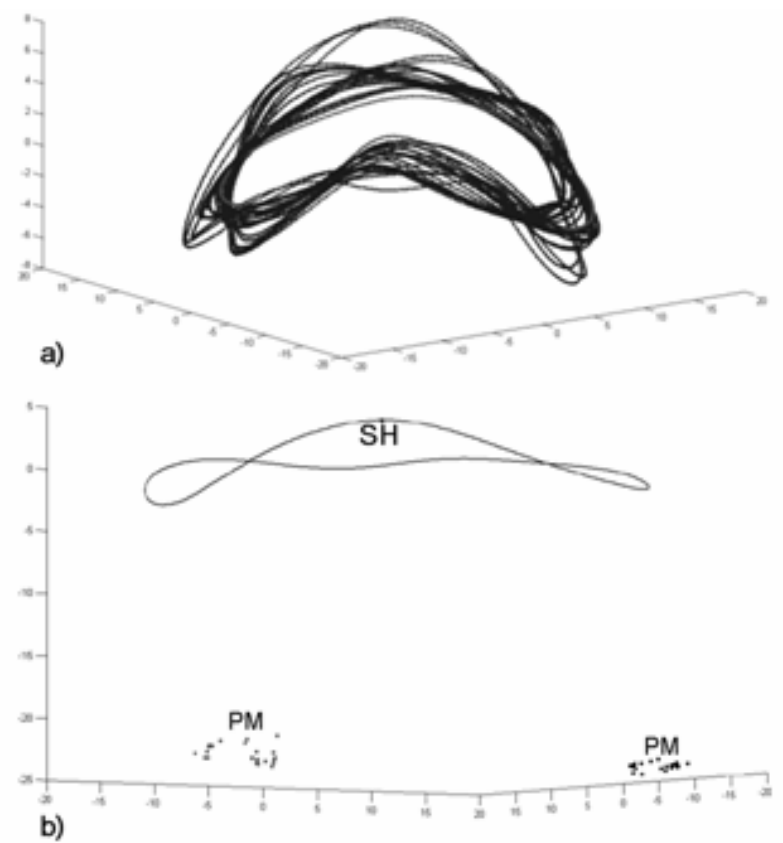

Figure 2: a) Reconstructed MA time-dependent profile; the ED configuration is depicted in black, subsequent configurations are depicted in grey. b) Reconstructed PMs position. For clarity's sake the ED configuration of the MA is also depicted ( $\mathrm{SH}=$ saddle horn). Axes values are in $\mathrm{mm}$.

\subsection{Tissues mechanical properties}

All tissues were assumed non-linear and elastic. Their mechanical response was described by means of proper strain energy potentials. Leaflets anisotropic response was accounted for by means of a Fung-like strain energy potential [3]. Chordae tendineae response was assumed isotropic. A second order polynomial strain energy potential was used for $1^{\text {st }}$ order chordae; a fifth order Ogden strain energy function was used for $2^{\text {nd }}$ and $3^{\text {rd }}$ order chordae. The constitutive parameters were defined via mean-square interpolation of data from the literature 
$[11,12]$.

\subsection{Boundary conditions}

The dynamic contraction of MA and PMs was modelled via kinematic boundary conditions, i.e. imposed as nodal displacements. A continuous MA profile was obtained from each RT3DE frame, interpolating the 36 tracked points. After sampling it into 404 nodes, nodal displacements with respect to the initial ED configuration were calculated. The motion of PMs tips was estimated from in vivo data obtained in animal models [13]. The time-dependent MA profile and PMs position are depicted in Figure 2.

To simulate the effect of blood pressure from ED to SP, a physiological time-dependent transvalvular pressure drop, up to $120 \mathrm{mmHg}$, was applied on the leaflets. Simulations were run using the ABAQUS/Explicit commercial software, version 6.7-1.

\section{Results and discussion}

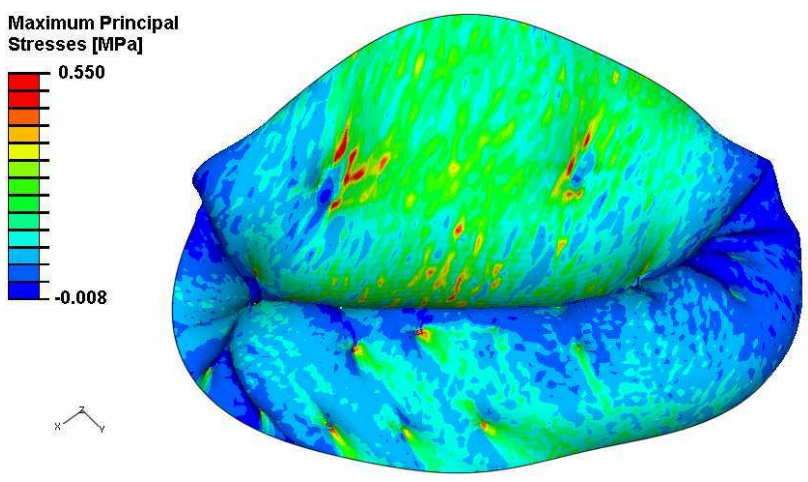

Figure 3: Leaflets maximum principal stresses at the systolic peak (atrial view).

Valve dynamics and leaflets coaptation were consistent with in-vitro observations: complete coaptation was obtained for a $18 \mathrm{mmHg}$ transmitral pressure [14] and, in accordance to clinical observations, the coaptation region corresponded to the leaflets rough zone.

The MV tensile state was analysed focusing on SP, as this timeframe is characterized by the maximum pressure load. Leaflets maximum principal stresses were computed (Figure 3). Their distribution reflected the asymmetry of the initial geometrical model. The anterior leaflet was more stressed than the posterior one, with peak values of $550 \mathrm{kPa}$ at the insertion of strut chordae.

Leaflets strains (Figure 4) reflected their anisotropic response, much stiffer in the direction parallel to the MA (top panel) than orthogonal to it (bottom panel). In particular, in the belly of the anterior leaflet, stretch ratios in the two directions were equal to 1.09 and 1.46 , in good agreement with experimental data [15].

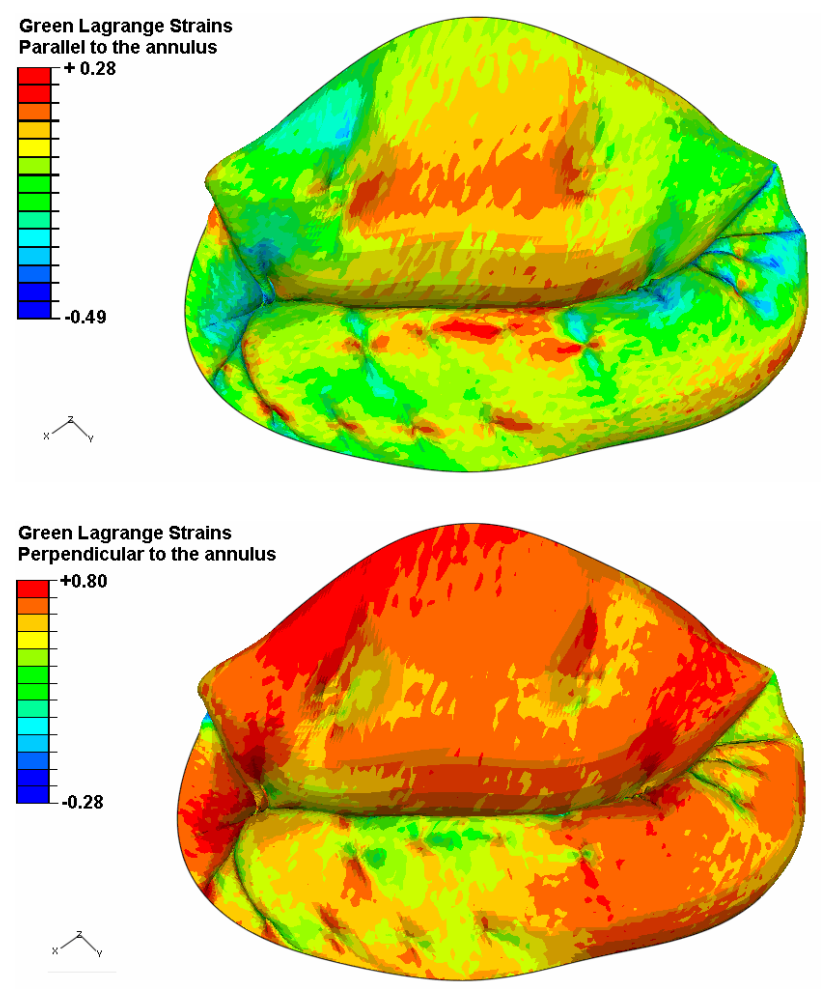

Figure 4: Leaflets Green-Lagrange Strains parallel to the annulus (top) and perpendicular to it (bottom).

Chordae tendineae tension for each type of chorda was monitored during valve closure (Figure 5 and Table 1). The average tension on a single chorda was the highest in strut chordae.

Table 1: Computed systolic peak chordae tensions, compared to in vitro data from literature.

\begin{tabular}{lll}
\hline Chordae Type & \multicolumn{2}{l}{ Systolic Peak Tension [N] } \\
\hline & FEM Data & In Vitro Data [15] \\
\hline Strut & $1.13 \pm 0.25$ & $1.11 \pm 0.57$ \\
Anterior Marginal & $0.13 \pm 0.04$ & $0.18 \pm 0.16$ \\
Posterior Marginal & $0.19 \pm 0.16$ & $0.08 \pm 0.11$ \\
Posterior 2nd Order & $0.12 \pm 0.10$ & $0.29 \pm 0.14$ \\
Commissural & $0.45 \pm 0.12$ & $0.40 \pm 0.31$ \\
Basal & $0.09 \pm 0.12$ & $0.48 \pm 0.25$ \\
\hline
\end{tabular}

However, marginal chordae as a whole bared the highest fraction of the entire load experienced by the chordal apparatus. Good agreement was found with experimental findings from the literature for the different chordae types [16]. 


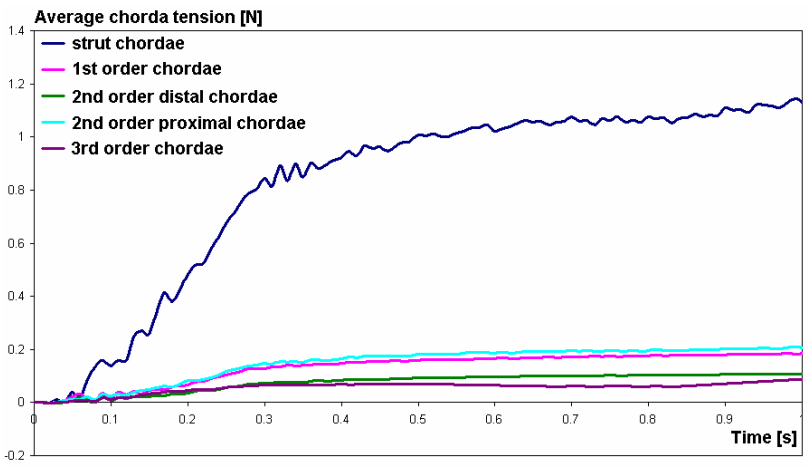

Figure 5: Time-course (from ED to PS) of chordae tendineae tension, averaged on chordae of the same class.

\section{Conclusions}

With this study, a novel approach to obtain a MV FEM with beyond-state-of-the-art features was defined and tested.

Results suggest that the use of RT3DE allows to overcome most of the limitations of previous MV models, thus obtaining a more realistic FEM. Although further testing on more subject-specific data is mandatory, this strategy may be at the basis for the development of a patient-specific modelling tool to be applied in the clinical settings or for surgical planning.

\section{Acknowledgements}

The research leading to these results has received funding from from the European Community's Seventh Framework Programme (FP7/2007-2013) under grant agreement $n^{\circ}$ FP7-224635 and from the Italian Ministry of Education, University and Research (PRIN 2007 funds, SurgAid Project).

\section{References}

[1] Kunzelman KS, Einstein DR, Cochran RP. Fluid-structure interaction models of the mitral valve: function in normal and pathological states. Philos. Trans R Soc Lond B Biol Sci 2007;362:1393-1406.

[2] Lim KH, Yeo JH, Duran CM. Three-dimensional asymmetrical modeling of the mitral valve: a finite element study with dynamic boundaries. J Heart Valve Dis 2005;14:386-392.

[3] Prot V, Haaverstad R, Skallerud B. Finite element analysis of the mitral apparatus: annulus shape effect and chordal force distribution. Biomech Model Mechanobiol 2008; Ahead of print.

[4] Cochran RP, Kunzelman KS. Effect of papillary muscle position on mitral valve function: relationship to homografts. Ann Thorac Surg 1998;66:S155-S161.

[5] Votta E, Maisano F, Soncini M, Redaelli A, Montevecchi FM, Alfieri O. 3-D computational analysis of the stress distribution on the leaflets after edge-to-edge repair of mitral regurgitation. J Heart Valve Dis 2002;11:810-822.

[6] Dal Pan F, Donzella G, Fucci C, Schreiber M. Structural effects of an innovative surgical technique to repair heart valve defects. J Biomech 2005;38:2460-2471.

[7] Votta E, Maisano F, Bolling SF, Alfieri O, Montevecchi FM, Redaelli A. The Geoform disease-specific annuloplasty system: a finite element study. Ann Thorac Surg 2007;84:92-101.

[8] Veronesi F, Corsi C, Sugeng L, Caiani EG, Weinert L, Mor-Avi V, Cerutti S, Lamberti C, Lang RM. Quantification of mitral apparatus dynamics in functional and ischemic mitral regurgitation using real-time 3dimensional echocardiography. J Am Soc Echocardiogr 2008;21:347-54.

[9] Kunzelman KS, Cochran RP, Verrier ED, Eberhart RC. Anatomic basis for mitral valve modelling. J. Heart Valve Dis 1994;3:491-496.

[10] Lam JHC, Ranganathan N, Silver MD. Morphology of the human mitral valve. I. Chordae tendinae: a new classification. Circulation 1970;41:449-458.

[11] Kunzelman KS, Cochran RP. Mechanical properties of basal and marginal mitral valve chordae tendineae. ASAIO Trans 1990;36:M405-M408.

[12] Liao J, Vesely I. A structural basis for the size-related mechanical properties of mitral valve chordae tendineae. J Biomech 2003;36:1125-1133.

[13] Dagum P, Timek TA, Green GR, Lai D, Daughters GT, Liang DH, Hayase M, Ingels NB, Miller DC. CoordinateFree analysis of mitral valve dynamics in normal and ischemic hearts. Circulation 2000;102:III62-69.

[14] Timek T, Glasson JR, Dagum P, Green GR, Nistal JF, Komeda M, Daughters GT, Bolger AF, Foppiano LE, Ingels NB Jr, Miller DC. Ring annuloplasty prevents delayed leaflet coaptation and mitral regurgitation during acute left ventricular ischemia. J Thorac Cardiovasc Surg 2000;119:774-783.

[15] Sacks MS, He Z, Baijens L, Wanant S, Shah P, Sugimoto H, Yoganathan AP. Surface strains in the anterior leaflet of the functioning mitral valve. Ann Biomed Eng 2002;30:1281-1290.

[16] Jimenez JH, Soerensen DD, He Z, Ritchie J, Yoganathan AP. Mitral valve function and chordal force distribution using a flexible annulus model: an in vitro study. Ann Biomed Eng 2005;33:557-566.

Address for correspondence

Dr Emiliano Votta

Dipartimento di Bioingegneria

Politecnico di Milano

Piazza Leonardo da Vinci 32, Milano, Italy

E-mail: votta@biomed.polimi.it 